
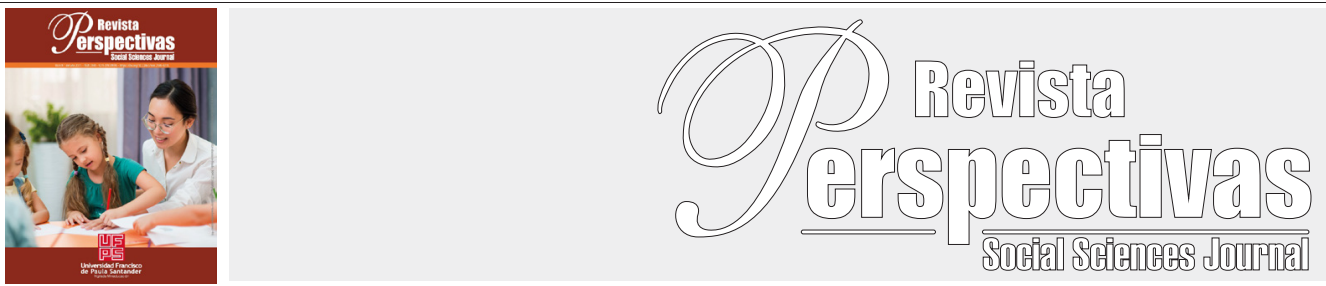

Original Article

https://doi.org/10.22463/25909215.2925

\title{
Movimientos sociales urbanos en Barcelona. \\ la prosperitat a través de las reivindicaciones ciudadanas y el derecho a la ciudad.
}

Urban social movements in Barcelona.

The Prosperitat through the citizens claims and the right to the city.

Erika Tatiana Ayala-Garcia ${ }^{1}$

${ }^{\prime}$ Doctora en Teoría e Historia de la Arquitectura, ORCID: https://orcid.org/0000-0001-7570-6639. erikatatianaayala@ufps.edu.co, Universidad Francisco de Paula Santander.

Como citar: E.T Ayala-García, "Movimientos sociales urbanos en Barcelona.la prosperitat a través de las reivindicaciones ciudadanas y el derecho a la ciudad.”. Perspectivas, vol. 6, no. 1,,pp. 72-84, 2021

Received: August 01, 2020; Approved: November 19, 2020.

\begin{tabular}{ll}
\hline Palabras clave: & RESUMEN \\
Barcelona, & El presente artículo de investigación habla sobre la importancia de los Movimientos Sociales Urbanos en \\
la ciudad de Barcelona, haciendo un énfasis especial en el barrio la Prospertitat del Distrito de Nou Barris; \\
Movimientos Sociales & el cual a partir de los años setenta a través de la Asociación de Vecinos desarrolló reivindicaciones urbanas \\
enfocadas al mejoramiento de la calidad de vida de sus habitantes en búsqueda de la adjudicación de nuevos \\
Sentido de Pertenencia,
\end{tabular}$\quad \begin{aligned} & \text { espacios públicos e infraestructuras de enfoque social. Su metodología es de enfoque cualitativo con una } \\
& \text { medición de dimensión no experimental, llevada a cabo bajo fuente de obtencion de datos documental y la } \\
& \text { Territorio, } \\
& \text { implementacion de entrevistas semiestructuradas realizadas a actores clave. Como hallazgos relevantes se } \\
& \text { destaca el papel de los Movimientos Sociales Urbanos con respecto a la reivindicación de demandas con } \\
& \text { relación a las falencias y desigualdades urbanas, los derechos sociales, económicos, culturales, previstas } \\
& \text { dentro del derecho a la ciudad. A su vez se resalta la fuerza y la cohesión vecinal presente en el barrio } \\
& \text { la Prosperitat con respecto a la solución de los problemas urbanísticos y sociales del mismo; logrando } \\
& \text { la adjudicacion de hitos conmemorativos y representativos como la Plaza Ángel Pestaña; y finalmente } \\
& \text { se determina la importancia de establecer dentro de la planificación urbana y territorial la participación } \\
& \text { ciudadana como mecanismo de innovacion social. }\end{aligned}$

\begin{tabular}{|c|c|}
\hline & ABSTRACT \\
\hline $\begin{array}{l}\text { Palabras clave: } \\
\text { Barcelona, } \\
\text { Urban Social Movements, } \\
\text { Sense of Belonging, } \\
\text { Territory, } \\
\text { Urbanism }\end{array}$ & $\begin{array}{l}\text { This research article studies the importance of Urban Social Movements in the city of Barcelona, with } \\
\text { special emphasis on the Prospertitat neighborhood of the Nou Barris District, which from the seventies } \\
\text { through the Neighborhood Association developed urban demands focused on improving the quality of life } \\
\text { of its inhabitants in search of the award of new public spaces and infrastructures with a social focus. Its } \\
\text { methodology is of a qualitative approach with a non-experimental dimension measurement, carried out under } \\
\text { a source of documentary data collection and the implementation of semi-structured interviews carried out } \\
\text { with key actors. As relevant findings, the role of Urban Social Movements is highlighted with respect to } \\
\text { the vindication of demands in relation to urban deficiencies and inequalities, social, economic and cultural } \\
\text { rights, provided for within the right to the city. In addition, the strength and neighborhood cohesion present } \\
\text { in the Prosperitat neighborhood is highlighted with respect to the solution of its urban and social problems; } \\
\text { achieving the award of commemorative and representative landmarks such as Plaza Ángel Pestaña; and } \\
\text { finally the importance of establishing citizen participation as a mechanism for social innovation is manifested } \\
\text { within urban and territorial planning. }\end{array}$ \\
\hline
\end{tabular}

\section{Introducción.}

Desde la perspectiva del urbanismo y la arquitectura, la gestión del territorio debe propender por la generación de estrategias, políticas, planes, proyectos y procesos que tengan como objetivo final el mejoramiento de la calidad de vida de los habitantes, a través de la satisfacción de las necesidades espaciales y funcionales tanto individuales como colectivas, así como la 
mitigación de problemáticas de orden socio-cultural, físico-ambiental y económico-normativo propias del contexto en el que se desarrollo o implanta.

Según autores como Ayala Garcia (2018) lo anterior se encuentra directamente relacionado con el concepto del derecho a la ciudad, al tener en cuenta que los territorios en los cuales se presentan asentamientos humanos (urbanos) densificados presentan complejidades derivadas de su función, su uso, sus temporalidades y sus dinámicas; Por lo tanto, estos territorios (ciudades) contienen aspectos tangibles e intangibles que deben ser diagnosticados, evaluados y analizados con el fin de que las respuestas otorgadas a sus problemáticas sean oportunas, coherentes y pertinentes. Al respecto, autores como Borja (2012 a.), establecen que "la ciudad del deseo no es la ciudad ideal, utópica y especulativa. Es una ciudad pensada, deseada e inventada, pero también es la ciudad resultante de aspiraciones, demandas de intereses y conflictos". Este planteamiento enfatiza la responsabilidad y el desafío cuantitativo y cualitativo que tiene la planeación urbana, a partir de tres frentes comunes que giran en torno a la población (habitantes): 1. los derechos y las obligaciones ciudadanas, 2. el acceso, la equidad y la igualdad en el territorio y 3. la promoción y el fortalecimiento de la ciudadanía (Social-cultural).

Bajo este orden de ideas, es importante reseñar que desde hace más 150 años las ciencias sociales han realizado estudios que abordan los Movimientos Sociales Urbanos (MSU); entendidos por Castells (2004), como una serie de acciones colectivas conscientes, a través de las cuales un grupo de personas pretende visibilizar y buscar solución a los diversos conflictos urbanos asociados a factores como el hábitat y la infraestructura, bajo un esquema comunitario. Desde este contexto, los MSU actúan partir de la experiencia cotidiana del ser individual y colectivo, ya que esta les permite interpretar la realidad territorial de un espacio y un contexto geográfico especifico, con el fin de generar una transformación o modificación urbano-territorial en un corto, mediano o largo plazo (Castells, 1972) apoyada en aspectos como la familiaridad, el sentido de pertenencia, el apego y el sentido de lugar; entre otros (Oslender, 2002).

En este sentido, para Magro Huertas (2009) los Movimientos Sociales Urbanos corresponden a la reunión de un grupo de personas pertenecientes a un territorio común, que promueven la denuncia y visibilización de las problemáticas y los conflictos urbanos, mediante la reivindicación de los derechos y las obligaciones ciudadanas, el acceso, la equidad y la igualdad en el territorio y finalmente, la promoción y el fortalecimiento de la ciudadanía (Ver figura 1).

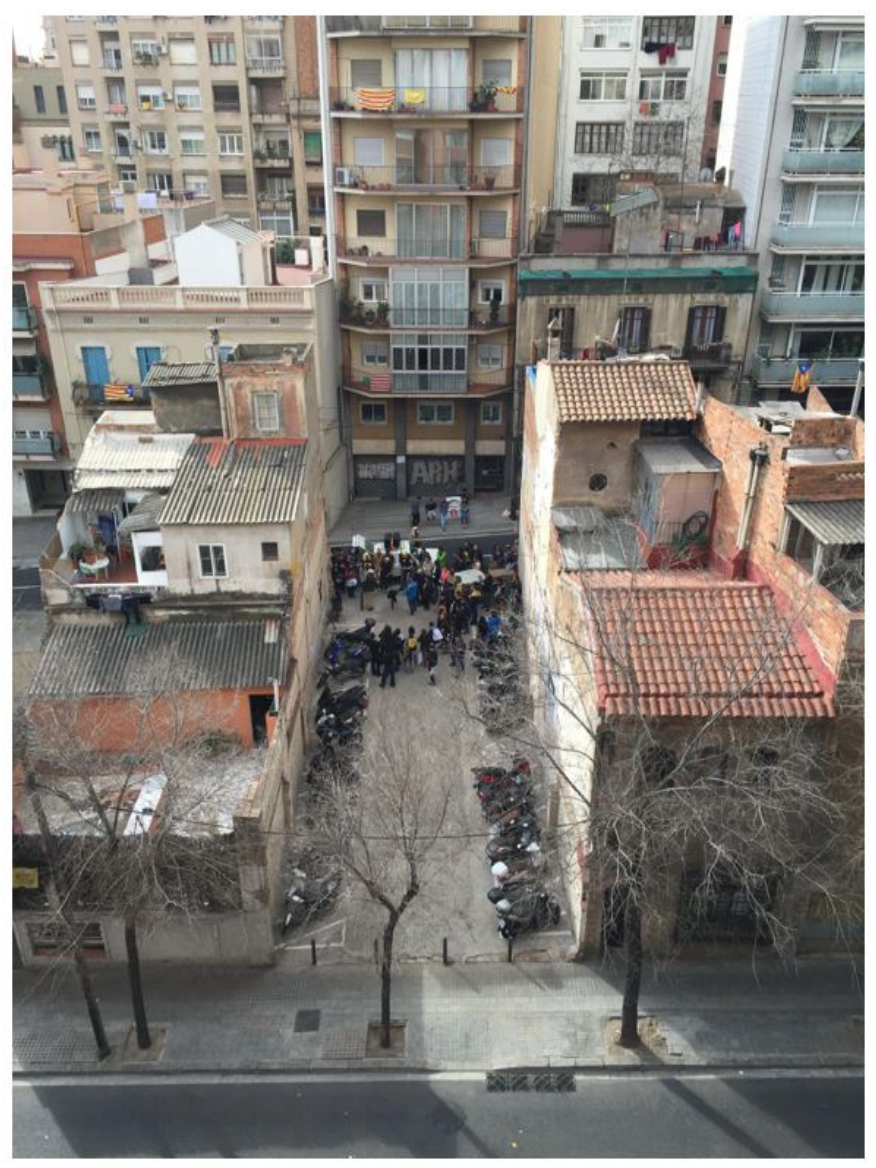

Figura 1. Movimiento Social en el barrio Vallcarca, Barcelona.

Fuente: Fotografía Arquitecto Juan Manuel Villa Carrero. Febrero 2016

Un ejemplo claro de lo anteriormente señalado corresponde a la ciudad de Barcelona (España), la cual a partir de los años treinta en respuesta al crecimiento acelerado de la población tuvo la necesidad de vincular núcleos urbanos aledaños 
a su territorio. Posteriormente en la década de los 60; frente a un nuevo crecimiento exponencial de la población y sumado a las consecuencias del fenómeno migratorio, la ciudad de Barcelona presento un déficit cualitativo y cuantitativo de vivienda y equipamientos que incidieron directamente en las características de habitabilidad, costo y, por lo tanto, en las condiciones de la calidad de vida de los habitantes (Oyon, 2002).

Desde esta perspectiva, autores como Andreu \& Huertas (1996) y Magro Huertas (2009) mencionan que los acontecimientos anteriormente señalados dieron lugar al nacimiento de los movimientos vecinales, los cuales fueron amparados por la Ley de Asociaciones de 1964 y la consolidación de las Asociaciones de Vecinos en 1966; hecho mediante el cual

"los vecinos junto al colegio de profesionales, algunos periodistas, partidos de izquierda, el COAC y la Oficina de Información Urbanista -OIU-, tomaron la vocería en relación a las problemáticas concretas que afectaban a los barrios (...) por medio de manifestaciones dentro del espacio público, la difusión a través de medios de comunicación como la prensa, y la creación de revistas por medio de las cuales se informaban de las luchas vecinales y los problemas de los barrios" (Ayala Garcia, 2018 pág. 66).

A partir de esto, en la Barcelona de los años 60 las problemáticas, inconformidades y necesidades urbanas fueron visibilizadas y expresadas mediante reivindicaciones sociales, por medio de las cuales los vecinos (habitantes) reconocieron y socializaron la situación, las particularidades y las deficiencias urbano-sociales de los barrios (Domingo \& Bonet, 1998). Este hecho trajo como consecuencia que el ciudadano se presentara como un agente activo dentro de la planificación, la gestión y la construcción de la ciudad.
En Revolución urbana y derechos ciudadanos: Claves para interpretar las contradicciones de la ciudad actual, Jordi Borja (2012 b.) recuerda que entre las décadas de los 70 y 90, las reivindicaciones sociales de Barcelona se centraron en la comprensión del territorio y la identificación de sus necesidades. Lo anterior, en palabras del autor trajo configuro la generación de una dinámica social, a través de la cual los habitantes establecieron "una política de hacer ciudad y ciudadanía" que permitió complementar las políticas publicas.

Así, para Borja (2012 b.) de la mano de los Movimientos Sociales Urbanos el espacio público cobra nuevamente importancia para el territorio, configurándose como el lugar y el medio propicio para la socialización, el conflicto, la reivindicación y la democracia, favoreciendo la promoción de demandas relacionadas con el hábitat, la vivienda, así como con los aspectos sociales, económicos, culturales y normativos contemplados en el derecho a la ciudad; recordando además, las palabras del Ministro Manuel Fraga en 1976 en las cuales declaró:

(...) "la calle es mía" y las asociaciones barriales de Barcelona y Madrid ocuparon la calle en los inicios del mes de febrero de 1976 (...) conquistando el espacio público incautado por la dictadura (...) Pág. 232.

\section{Material y método.}

La presente investigación se realizó a partir de un enfoque cualitativo, que tiene como objetivo según autores como Hernández Sampieri (2014) la recolección y el analisis de datos, mediante la implementación de herramientas e instrumentos indagatorios llevados a cabo a traves de procesos logicos que favorezcan la exploración, descripción, interpretación y por ende la formulación de perspectivas teoricas. Asimismo, esta investigación presenta una medición de dimensión no experimental a traves de la cual se observan y analizan sin manipulacion de variables fenomemos o situaciones ya existentes que permiten llevar a cabo un análisis 
descriptivo en el cual se establecen las caracteristicas, fenomenos o sucesos propios de un grupo de personas o una comunidad (Tamayo y Tamayo, 2004).

$\mathrm{Su}$ obtencion de datos es documental, desarrollada bajo tecnica de analisis de contenido y sistematización de recursos e investigaciones cientificas (Peña Vera \& Pirela Morillo, 2007) derivadas de libros, tesis doctorales, de maestría y artículos de bases de datos bibliográficas como Scopus, Mendeley, Science Direct y Dialnet; entre otros, debidamente categorizados y analizados.

La investigación implementa la entrevista semiestructurada como instrumento de recolección de información. Estas fueron desarrolladas a actores claves durante el periodo 2008- 2016 tales como Maruja Ruiz, representante del Casal per la Gent Gran de la Prosperitat y lider vecinal, Albert Recio, exmiembro y líder de la asociación de vecinos de la Prosperitat y la arquitecta Benedetta Tagliabue de la Fundación Enric Miralles. Es de resaltar que las personas anteriormente mencionadas, cumplieron un papel fundamental durante los hechos analizados dentro de la presente investigación. Finalmente se utiliza soporte fotografico imágenes de cortesia o derivadas del Arxiu Històric de Roquetes- Nou Barris.

\section{Resultados y discusión.}

El distrito de Nou Barris: Se encuentra ubicado al norte de la ciudad de Barcelona, entre la avenida Meridiana y la sierra de Collserola, y está compuesto por trece barrios (Ver figura 2). Según datos ofrecidos por el Ayuntamiento de Barcelona, Nou Barris era el nombre de la revista de la primera Asociación de Vecinos de la zona durante los años setenta, y fue ese el nombre adoptado para el distrito número ocho, a partir de la división de la ciudad en diez distritos, realizada en 1984.

Una de las características que llama la atención, respecto a este distrito se encuentra representada a través de la composición y el paisajismo rural que lo precedía hasta el primer tercio del siglo XIX, hecho que cambió radicalmente a partir del siglo XX de la mano de los nuevos desarrollos urbanísticos (Ayuntamiento de Barcelona, S.F).

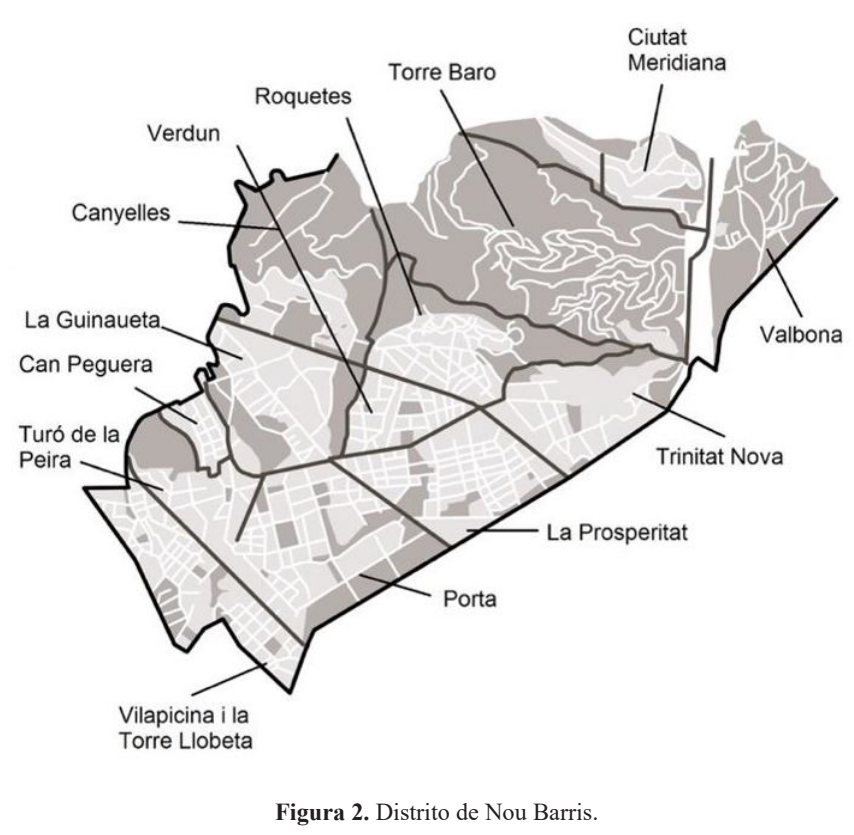

Fuente: María Daniela Tami Cortes a partir del Ayuntamiento de Barcelona (S.F).

Según autores como García Soler (1998), a mediados del siglo XIX las características de la vida rural en torno a Barcelona comienzan a sufrir un cambio derivado de la Revolución Industrial, como respuesta al crecimiento acelerado de la población; la revolución del transporte y los nuevos procesos de industrialización, sumado al crecimiento dinámico que experimentó Nou Barris hacia el año 1936, que dio origen a barrios como Roquetes, Verdum, la Prosperitat y la Guinaueta, entre otros (Martínez Fernández, 1982).

El barrio de la Prosperitat según datos de lectura del Ayuntamiento de Barcelona, en el año 2020 contaba con 26.166 habitantes, distribuidos en una superficie 59, 50 (ha) y una densidad poblacional de $444 \mathrm{hab}$./ha. Este barrio se caracteriza por la presencia de construcciones que datan del comienzo del siglo, así como por un número elevado de edificaciones levantadas al largo de la segunda mitad del siglo XX. 
La Prosperitat a lo largo de los años se ha distinguido por su historia y por la tenacidad de sus habitantes, quienes han debido luchar a través de la Asociación de Vecinos contra problemáticas urbanas claras tales como la alta densidad de urbanización y la falta de planificación. Hechos que se han visto reflejados en las necesidades básicas del barrio como la falta de adjudicación y construcción de espacios libres y aparcamientos subterráneos, sumados a la escasa inversión pública en sectores como equipamientos, servicios y educación (Ayala Garcia, 2013, págs. 62-65).

Desde 1974 los vecinos del barrio conscientes de las necesidades de este desarrollaron reivindicaciones y protestas en contra del Ayuntamiento, con el fin de obtener equipamientos y espacios públicos de calidad como la Plaza Ángel Pestaña, la plaza Harry Walker, el Casal de barrio, el Casal de jóvenes, el Casal del adulto mayor y el Ateneo; entre otros (Ayala Garcia, 2013) minimizando el impacto de afectaciones sociales y urbanas.

Según el Centre de Documentació i estudis dels Nou Barris (1985), entre los años 17901980 el crecimiento de la población del barrio fue de un $17,5 \%$, mientras que paralelamente el parque inmobiliario aumentó en un 30,35\%. Esta densificación no sólo se tradujo en la escasez de espacios construidos, sino en la reducción de las dimensiones de estos, hecho que se vio agravado por la falta de solares destinados a dar soporte físico a las necesidades de equipamientos y zonas verdes de barrio.

Tengo cincuenta años viviendo en el barrio, bueno en los movimientos sociales tengo cincuenta años, mira mi experiencia es el llegar a este barrio donde no habia nada y empezar a luchar, no habia colegios, no había calles asfaltadas, no había nada (...)

Ha sido una lucha constante, por este casal (Casal de Gent Gran) estuvimos 17 años luchando sin parar y por esa plaza (plaza Ángel Pestaña) diez (...) que estuvimos diecisiete años luchando por este casal, yo era de la Asociación de Vecinos y entonces aqui había un edificio viejo, querían edificarlo y como era pequeño el Ayuntamiento vendió la calle Casanovas. ¡La calle no se puede vender! no es de ellos. Por eso cuando vino el Puyol le dije "vienes con diecisiete años de retraso" y luego la constructora dijo que nos hacía un puente y le dijimos que no, y entonces mientras tanto hubo un plan comarcal y se aprobó que fuera equipamiento... y por un semáforo luchamos 21 días, cortando el tráfico. Todo ha costado mucho tiempo.

(...) en Torre Baró no iba el transporte, y vivían dos mil personas y le habíamos pedido que, porque no podía ir, el Ayuntamiento decía que no le era rentable para dos mil personas y que lo del transporte no era posible, hasta que un día y ya después de mucho, de no tener más posibilidad me fui a Torre Baró hice una asamblea con la gente y les dije "si vosotros queréis que comprobemos o no, mañana a tal hora a la plaza Llucmallor. Entonces llegando a la parada del bus le dije al conductor, jeste va a Torre Baró! y me dijo que no, jentonces le dije pues bájate que lo llevo yo! Cogimos el autobús y fuimos a la plaza de Torre Baró, y al llegar allá llamé a los medios de comunicación y les dije "no ha pasado nada, el autobús ha llegado" y a partir de ahi tienen transporte (...).

\section{Maruja Ruiz, Casal per la Gent Gran la Prosperitat.}

En el barrio de la Prosperitat la lucha ha sido constante, intensa y duradera; el sentido de pertenencia de sus habitantes los ha llevado a levantar su voz con el fin de satisfacer sus necesidades. Una plaza, un parking, un casal, son algunas de las preocupaciones de un barrio que a pesar de sus continuas reivindicaciones se encuentra representado por 5 bibliotecas públicas (Nou Barris) de las 36 que se encuentran en la ciudad de Barcelona, así como por la ausencia de bibliotecas especializadas, 
generales, archivos, museos y teatros, y la falta de equipamientos destinados al mejoramiento de la calidad de vida de los jóvenes y las personas mayores, que como se mencionó con anterioridad representan una parte importante de su población.

La plaza Ángel Pestaña: Según el informe denominado “La plaça d’Àngel Pestaña, el cor del barrí de la Prosperitat", realizado por San Nicolás y Rodríguez (2016), a lo largo del tiempo las luchas vecinales han caracterizado y marcado a Nou Barris, recordando a través de la voz de sus habitantes, como es el caso de Andrés Naya, miembro de la Asociación de Vecinos, que espacios como la plaza Ángel Pestaña (urbanizada en 1986) han sido el resultado de múltiples reivindicaciones vecinales en pro del mejoramiento de la calidad de vida del barrio.

Dicho estudio corrobora que antes de la construcción de la plaza el espacio era conocido como las barracas de Santa Engracia, resultado de una estafa inmobiliaria a principios de los sesenta en la que decenas de familias afectadas por la construcción inconclusa de sus viviendas se instalaron en el plano de Santa Engracia, entre las calles Casanovas y San Francisco Javier, bajo unas condiciones muy precarias, carentes de servicios de agua y luz, hasta 1983 donde fueron trasladados a la viviendas de Renfe- Meridiana (Ver figura 3).

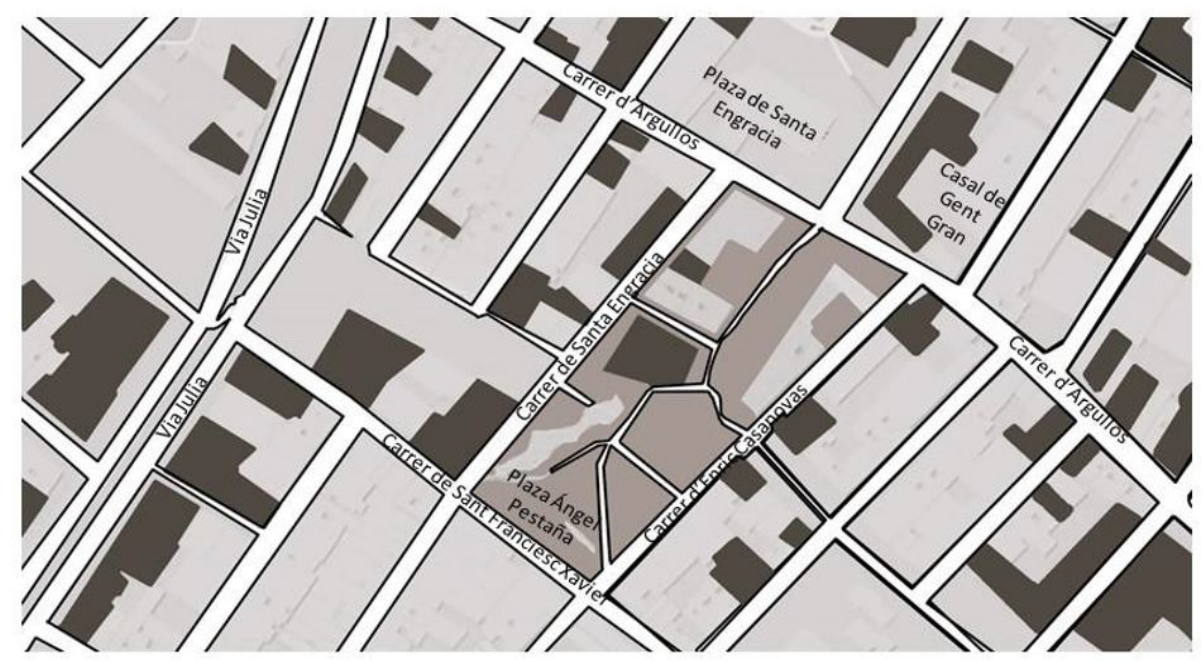

Figura 3. Ubicación Plaza Ángel Pestaña

Fuente: María Daniela Tami Cortes (2018).

Las condiciones insalubres a las que estaban sometidos los habitantes de las barracas generaron inconformidad en la comunidad (Ver figura 4), ocasionando falsas alarmas sobre focos infecciosos de brotes de meningitis y tuberculosis, hecho que trajo como consecuencia el rechazo de los vecinos del barrio hacia los habitantes de las barracas.

Con base en esto, Albert Recio, exmiembro y líder de la Asociación de Vecinos en Ayala Garcia (2018 págs. 123-124) menciona que:
(...) Bueno, te cuento, ósea el origen de la plaza, tú has de cuenta esta es una zona de población, básicamente inmigración a la ciudad en los años 50 o 60, que en un modelo de desarrollo donde no había ninguna inversión de infraestructura pública, con muy poco gasto público y donde esto genera un movimiento vecinal muy potente en toda la zona (...)

En concreto donde está la plaza Ángel Pestaña. Donde antes era una zona de barracas, que eran unas barracas que se habian creado a partir de un 
negocio inmobiliario que habían vendido pisos y había fracasado. Y entonces en el inicio la gente ocupó la zona de barracas, y este era un tema históricamente complicado, porque en las barracas lo que más había era población de gitanos, no todos (...) Habia unas condiciones de vida muy malas de los barraquistas, y después había también una parte de racismo por los vecinos. Y entre los vecinos la lucha era conseguir el espacio aquel, para espacio público. Y después conseguir viviendas dignas para la gente que vivía ahí.

Esto se consigue claro... no se los años exactos, sobre los ochenta, mitades de los ochenta. Conseguimos que cerca, no en el mismo barrio, pero al lado de otro barrio José Valldaura, con una lucha muy importante donde prácticamente se salvan para la ciudad los espacios que ocupaban los talleres del ferrocarril, de la RENFE. Una parte de este espacio que era muy grande se dedica a pisos, y se mueven hacia estos pisos la gente que vivía en las barracas y otra gente afectada (...)

Albert Recio, expresidente Asociación de vecinos de la Prosperitat.

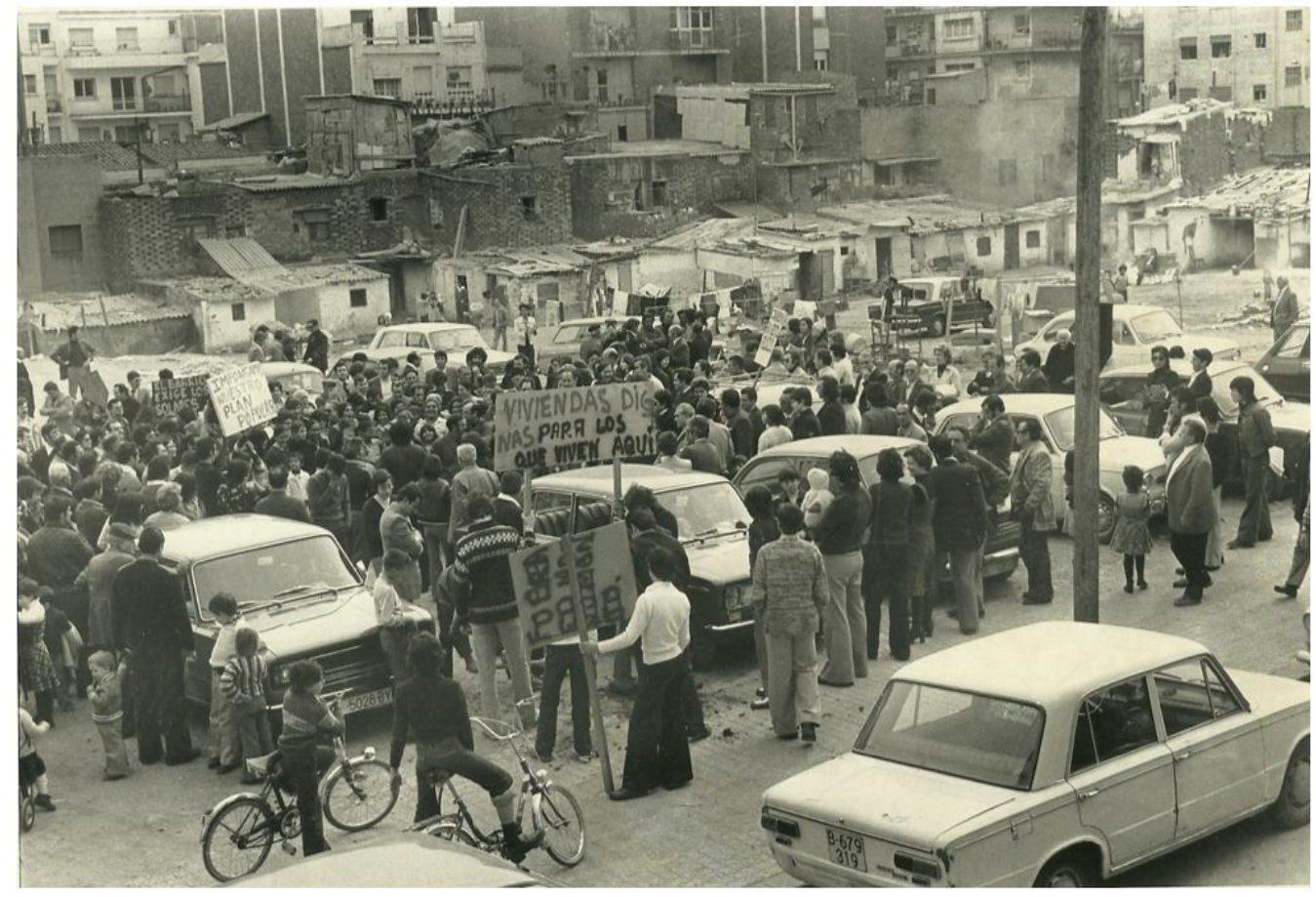

Figura 4. Barracas de Santa Engracia F (10-1339).

Fuente: Arxiu Històric de Roquetes- Nou Barris.

Los habitantes del sector representados en la Asociación de Vecinos realizaron protestas encaminadas al desarrollo de nuevas estrategias urbanas que favorecieron el desarrollo del barrio como la creación de espacios públicos de carácter temporal, arborizados, equipados y amoblados, con condiciones que fomentaran el paseo, el disfrute, la seguridad y la accesibilidad (Del Cas, Gigoso, \& Saravia, 2002). Es decir, espacios con las características necesarias para el fomento de la vida en la calle, la vida pública, la vida en el barrio (Ayala Garcia, 2013, pág. 66).

En este sentido, la arquitecta Benedetta Tagliabue en Ayala Garcia (2018) recuerda el proceso vivido durante la adjudicación y construcción de la plaza Ángel Pestaña, resaltando la fuerza y vitalidad de los habitantes del barrio, quienes se convirtieron en una de las principales fuentes de inspiración para su equipo de trabajo. Tagliabue recuerda que 
el proyecto se desarrolló en una época donde el urbanismo estaba en auge en Barcelona y su equipo de trabajo se encontraba en proceso de afianzamiento debido a la enfermedad y posterior muerte del arquitecto Enric Miralles.

“(...) Yo creo que esta plaza tiene una historia un poco especial, fue un encargo que recibimos en el año 2000 de parte de un político que ahora ya no me acuerdo... Era un político que en esa época era muy influyente en la parte de urbanismo de toda Barcelona (...) definimos este encargo, pero no tuvimos ni tiempo de empezar porque poco después Enric se dio cuenta que estaba enfermo, nos fuimos a estados unidos a Houston y desde allí Enric pensaba en los proyectos estando en contacto con el estudio, (...) por ese proyecto teníamos que hacer una pequeña entrega y Enric redactó un texto.

Este texto (el de la plaza Ángel Pestaña), era un texto muy en relación a la vida, porque él se daba cuenta en ese momento que vivir era disfrutar de la calle o disfrutar de un clima como el de Barcelona, estar con la posibilidad de entrar a un bar (...) y el texto un poco recoge eso, porque la ciudad y el barrio son fantásticos cuando se viven de esta manera (...) fue uno de los últimos que escribió el de su puño, llegó aquí al estudio lo transcribieron (y creo que está transcrito seguramente lo podremos encontrar) pero el proyecto lo hicimos ya después, cuando él estaba muerto (...)

\section{Benedetta Tagliabue.}

El proyecto de la Plaza Ángel Pestaña nace entonces como respuesta a las reivindicaciones generadas por los MSU de los vecinos de la Prosperitat, siendo un proyecto adjudicado al arquitecto Enric Miralles, quien para cumplir con su primera entrega redactó un escrito que condensa las características vivenciales que configuraron el proceso exploratorio de la plaza y que finalmente soportaron la morfología de la misma; variantes que sin lugar a duda representan una armonía y un equilibrio claro respecto a la cotidianidad del barrio y a las características de sus habitantes, resaltando a nivel morfológico la vida, la intensidad, el movimiento y la cohesión que caracteriza La Prosperitat.

\section{LA CALLE PABLO IGLESIAS NO ES UNA CALLE, ES UN LUGAR.}

Un lugar que nos permite jugar con el tiempo.

Tengo la sensación de que Nou Barris necesita dejar pasar el tiempo sobre sus calles rehechas tantas veces. Esta sensación la he tenido en los caminos de la Acrópolis de Atenas, en Pompeya, en lugares donde el tiempo ha ido acompañando la vida de la gente.

Imagino que esta cota horizontal es un lugar privilegiado... pasear arriba y abajo. Volver a encontrar un ritmo que Nou Barris había tenido.

Habéis hecho grandes trabajos, de limpieza, trazados, urbanización.

Ahora os propongo tomar este sitio, esta calle, y tratarlo como un punto excepcional de Barcelona.

HABÉIS DESCUBIERTO ESTE PASO ENTRE CALLES POR PRIMERA VEZ. ¡HABÉIS DESCUBIERTO UN LUGAR!

Con la mirada lo habéis sacado a la luz con todos sus rincones. Las posibilidades se multiplican. Es fácil imaginar un valle por donde encontrar los mejores lugares de paso.

Los problemas concretos se transforman en la parte divertida del trabajo: Aprovechar los problemas, no hacerlos desaparecer, Los desniveles, una puerta, un garaje.

No unificarlo todo demasiado, no tener miedo de las excepciones, estar contentos con la sorpresa. 
ESTA CALLE CONSTRUIRÁ UN ESPACIO URBANO INTERIOR, no es sólo un lugar de paso. No os preocupéis, habrá aparcamientos, vados, entradas, servicios, bancos, papeleras, árboles... pero de una calidad especial.

La gente, cuando esté construido. Se encontrará con la Calle, ENCONTRARÁ UN LUGAR PARA SENTARSE, ENCONTRARÁ UNA SOMBRAQUELE PERMITA IMAGINAR DÓNDE SE ENCUENTRA.

Más técnicamente esto se dice un lugar sin jerarquía, un espacio público abierto en el que los papeles cambian: es el ideal del espacio libre que permite muchos tipos de comportamiento.

Eso lo he experimentado en otros proyectos en más o menos grados de libertad. En el parque de Mollet del Vallès y en el de Diagonal Mar. Pero en esta Calle el problema es muy claro: mantener las dimensiones, las PEQUEÑAS VARIACIONES DE LA COTA HORIZONTAL HASTA CONSTRUIR UN PAISAJE HORIZONTAL, UN LUGAR DONDE VALGA LA PENA PONER LOS PIES EN EL SUELO.

QUE SALIR A LA CALLE SEA COMO LEVANTARSE POR LA MAÑANA, QUE ESTA CALLE SEA LO MÁS DESPIERTO.

Para terminar, dejarme hablar del arquitecto griego PYKIONIS. Es un personaje enigmático, sabe hacer proyectos sin hablar del problema. Los pavimentos de la Acrópolis son una lección de respeto. Hay un momento magnífico:

Un punto que nos hace alejarnos andando hasta dejar las piedras y donde un banco nos hace ver la distancia donde estamos. La Acrópolis aparece delante nuestro en Su solitud en el tiempo, Delicadamente.

Muchas veces se han hecho obras de excesiva dureza: automáticas en el espacio urbano, y la solución no son pequeñas islas verdes.
Creo que tenemos que trabajar viendo las posibilidades de transformación de este lugar, las líneas invisibles que esperan salir a la calle y que construirán una red de CAMINOS INVISIBLES que transformarán este espacio público en un espacio interior, DONDE VALGA LA PENA DESPERTARSE POR LA MAÑANA Y SALIR A TOMAR UN CAFÈ $A L B A R$.

Enric Miralles, primera presentación proyecto Plaza Ángel Pestaña. Mayo, 2000.

Para la arquitecta Tagliabue la Plaza Ángel Pestaña manifiesta una fuerte e intensa relación con los vecinos del barrio, la idea plasmada por Enric Miralles consistía en diseñar un espacio multifuncional en el que los vecinos pudieran realizar diversas actividades, generando lazos de pertenencia e identidad, utilizando y reforzando las características morfológicas del espacio.

(...) el barrio tiene mucha fuerza con gente organizada que nos decía lo que quería y lo que no quería, y nosotros siempre nos hemos divertido, siempre nos ha gustado escuchar las preguntas, lo que quieren ellos (...) y tienen un casal enorme, fueron ellos con su fuerza que lo consiguieron y son felices, esto me gusta. Hace diez años que no voy.

Es muy bonito que los vecinos sean tan orgullosos, es una de las cosas más bonitas de haber trabajado en Nou Barris. (...) Las graderías eran un intento de transformar un espacio natural pero también en un teatro (...)

\section{Benedetta Tagliabue.}

Asimismo, la arquitecta enfatiza que el proyecto de la Plaza Ángel Pestaña tuvo como marco de referencia el proyecto del Parque de Los Colores realizado entre los años 1992 y 2002, un espacio de crecimiento para la firma Miralles-Tagliabue que le permitió explorar tanto morfológica como vivencialmente, enriqueciendo considerablemente 
sus proyectos, sirviendo de fuente de inspiración para el desarrollo de la plaza Ángel Pestaña, por medio de la utilización de la morfología como componente que da fuerza y vitaliza el proyecto se convirtió en el punto más fuerte de estos proyectos.

En el caso de la plaza Ángel Pestaña la integración del componente natural (morfología) con la fuerza y el dinamismo de los vecinos del barrio, logró establecer lo que en palabras del arquitecto Miralles se puede definir como "un lugar donde valga la pena poner los pies en el suelo. Que salir a la calle sea como levantarse por la mañana, que esta calle sea lo más despierto”.

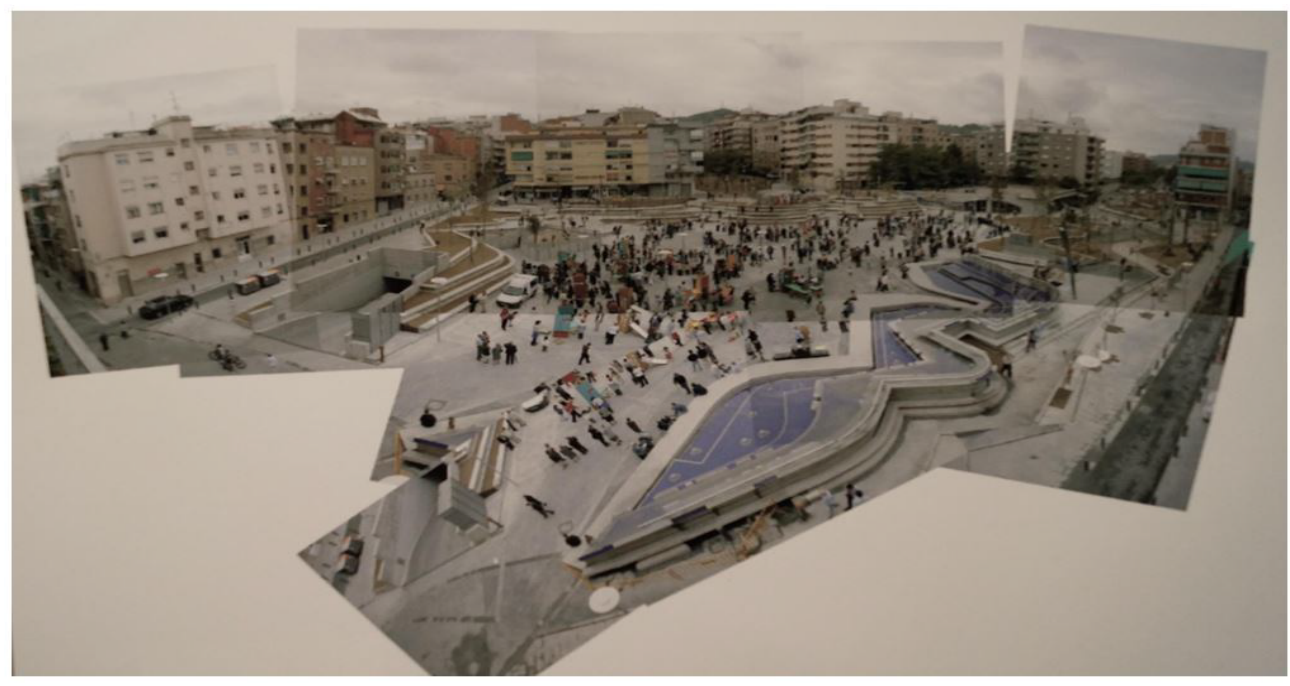

Figura 5. Montaje fotográfico Plaza Ángel Pestaña.

Fuente: Imagen cortesía para el trabajo de campo de la Tesis Doctoral realizado en la Fundación Enric Miralles, enero 2015.

Como se puede apreciar en la Figura 5 la utilización del pavimento dentro del proyecto obedece a la generación de espacios multifuncionales que favorecen el desarrollo de actividades. Igualmente, las ondulaciones de las bancas representan la búsqueda formal de un escenario teatral que se nutre a partir de las formas naturales del terreno y ofrece al usuario la posibilidad de establecer espacios de observación y participación comunitaria. Finalmente, la implementación de juegos infantiles como parte del pavimento denotan una invitación al colectivo infantil para hacer presencia constante dentro de la plaza.

Para los vecinos de la Prosperitat los diversos procesos desarrollados en la plaza Ángel Pestaña han traído como consecuencia aspectos positivos a nivel de cohesión vecinal, asumiendo positivamente los cambios y la evolución a los que ha estado sujeto este espacio con el paso de los años, teniendo en cuenta que dentro del barrio se ha fortalecido como un espacio de integración y encuentro comunitario; de corte social, a través del cual se mantiene latente la historia del barrio. Esta plaza ha logrado configurarse como uno de los espacios multifuncionales más representativos dentro de la Prosperitat. En ella tienen lugar diferentes actividades llevadas a cabo a lo largo de las temporadas del año tales como el Prospebeach, el festival de San Xiveco, las Fiestas Mayores del barrio, temporadas de cine a la fresca; entre otras, que, unidas a la presencia diaria por parte de los habitantes, logran que su utilización sea constante y permanente. El emplazamiento de la plaza y el diseño o la morfología de esta favorecen la apropiación de la misma por parte de los diferentes colectivos sociales que conforman el barrio; determinando así un escenario material físico- construido en el cual se 
ve representada y materializada la actividad social y la cohesión barrial.

Con respecto a lo anterior, autores como Guevara Salamanca, Hernández García \& Mendoza Molina (2011) aseguran que la idea de comunidad se encuentra directamente relacionada con el concepto y la percepción que manifiestan los habitantes respecto al territorio en el que se desenvuelven, promoviendo bajo conceptualizaciones positivas sentimientos de arraigo derivados de las tradiciones y las dinámicas sociales presentadas a lo largo de los años. De esta manera, el territorio se fortalece a través de los diferentes significados asociados al mismo como producto de las manifestaciones de la vida social, siendo entonces los lugares testigos $y$ actores de la vida de sus habitantes, al consolidar tradiciones, narrar historias y construir memoria.

\section{Conclusiones}

En la presente investigación se determinó que en Barcelona los Movimientos Sociales Urbanos se han configurado como el medio a través del cual los habitantes o vecinos de un barrio, han obtenido adelantos o mejoras significativas en relación a la satisfacción de las necesidades o problemáticas de su contexto inmediato, mediante la generación de una identidad colectiva, que les permite llevar a cabo acciones para el reconocimiento de las debilidades y fortalezas del territorio, seguidas de reivindicaciones sociales por medio de las cuales se busca la satisfacción de las necesidades comunitarias o colectivas.

De esta manera, se destaca la trayectoria y el papel desempeñado por los miembros de la Asociación de Vecinos del Barrio de la Prosperitat, al tener en cuenta que mediante la organización consiente y el entendimiento de su espacio físicoconstruido, los vecinos lograron establecer las problemáticas urbanas mas representativas del barrio, visibilizando y gestionando ante las autoridades competentes la solución de estas. Es de resaltar además como a partir de este proceso, el barrio de la
Prosperitat logró fortalecer la imagen y la identidad colectiva del barrio; estableciendo dinámicas asociadas a la memoria, el arraigo, la identidad y el sentido de pertenencia de la comunidad. Por lo tanto, los cambios gestados a partir de los MSU del barrio de la Prosperitat de Barcelona no solo mejoraron las problemáticas de carácter físicoconstruido del barrio y la calidad de vida de sus habitantes, sino que fortalecieron el aspecto sociocultural de la población a través de la humanización de los espacios, la transmisión de las tradiciones de generación en generación y la cohesión barrial; potencializando así las características tangibles e intangibles que configuran la Prosperitat como un espacio único y extraordinario.

Así, queda en evidencia la importancia que, dentro de los procesos de planeación urbana $\mathrm{y}$ territorial, se realicen procesos de reconocimiento de los territorios, logrando desarrollar estudios en los cuales la población pueda llegar a otorgar aportes significativos con respecto al reconocimiento de las necesidades físicas y vivenciales que las mismas manifiestan con respecto a su realidad y cotidianidad. De esta manera, los procesos de planeación y desarrollo urbano pueden llegar a establecerse como soportes de innovación social en donde la arquitectura actué como puente y satisfactor de las necesidades de quienes lo utilizan.

En el caso del barrio de la Prosperitat, los procesos llevados a cabo por la comunidad de la mano de los MSU han realizado aportes que han fortalecido el aspecto material y vivencial del barrio a lo largo de los años, el logro significativo de la obtención de los espacios públicos y de prestación de servicio social como los casals y las plazas; entre otros, sumados a la fuerza y la cohesión de los habitantes del barrio, establecen la Prosperitat como un espacio vivencial unificado, en el cual su población durante su historia de vida ha utilizado, apropiado y transformado sus espacios físico-construidos, dotándolos de vivencialidad, identidad, apego y 
memoria, generando a su vez relatos e historias que se transmiten de generación en generación.

\section{Referencias}

Andreu, M. \& Huertas, J. M. (1996). Barcelona en 1luita. El movimient urbà 1965-1966. Barcelona: Federació d'Associacions de veins de Barcelona FAVB.

Ayala Garcia, E. T. (2013). Territorialidad y reivindicación social: Reflexiones en torno al barrio La Prosperitat/Barcelona desde la perspectiva del adulto mayor. Educación y humanismo, 15(25), 59-72.

Ayala Garcia, E. T (2018). El espacio público desde la perspectiva del colectivo infantil y el de los adultos mayores. Un estudio interdisciplinar entre la arquitectura y las ciencias sociales. Tesis Doctoral. Barcelona: Universidad Politécnica de Cataluña.

Ayuntamiento de Barcelona (S.F). Breu història de Nou Barris. Recorregut pel paisatge i la identitat de Nou Barris. Disponible en: http://lameva. barcelona.cat/noubarris/ca/historia-del-districte)

Borja, J. (2012 a.). Revolución urbana y derechos ciudadanos: Claves para interpretar las contradicciones de la ciudad actual. Barcelona: Universidad de Barcelona.

Borja, J. (2012 b.). Movimiento ciudadano en busca de la ciudad futura (frente a la ciudad disuelta y la izquierda errante). En V. Pérez Quintana, \& P. Sánchez León, Memoria ciudadana y movimiento vecinal. Madrid, 1968-2008. Catarata.

Castells, M. (1972). La cuestión urbana. Madrid: Siglo XXI.

Castells, M. (2004). La cuestión urbana. Siglo XXI de España.
Del Cas, R. Gigoso, P. \& Saravia, M. (2002). La ciudad y los derechos humanos. Una modesta proposición sobre derechos humanos y práctica urbanística. Madrid: Talasa.

Domingo, M., \& Bonet, M. R. (1998). Urbanisme i participació. Revista Catalana de sociología (7).

Garcia Soler, C. (1998). Nou Barris. Barcelona: Centre d'Estudis i Documentació de Nou Barris.

Guevara Salamanca, J. D; Hernández García M \& Mendoza Molina, M (2011). Construcción y significación del territorio Comunidad el Codito. Bogotá: Universidad del Rosario.

Hernández Sampieri, R. (2014). Metodología de la investigación. 6 ed. México: Mc Graw Hill.

Magro Huertas, T. (2009). La espuma que golpea las puertas de la ciudad. Movimientos Sociales Urbanos en Barcelona (1964-1986). Barcelona.

Martinez Fernandez, F. (1982). Conoguem el nostre barri, la Prosperitat. Guia didactica i activitats. Barcelona: Centro de documentació i estudis dels Nou Barris. Associació de veins i veines de la Prosperitat.

Oslender, U. (2002). Espacio, lugar y movimientos sociales: Hacia una espacialidad de resistencia. Scripta Nova, VI. Universitat de Barcelona.

Oyon, J. L. (2002). Historia urbana e historia obrera: Reflexiones sobre la vida obrera y su inscripción en el espacio urbano 1900-1950. Perspectivas urbanas (2).

Oyon, J. (2004). La quiebra de la ciudad popular. Espacio urbano, inmigración y anarquismo en la Barcelona de entre guerras 1914-1936. Barcelona: Ediciones Serbal

Peña Vera, T. \& Pirela Morillo, J., 2007. La 
complejidad del análisis documental. Información, cultura y sociedad: revista del Instituto de Investigaciones Bibliotecológicas, Issue 16, pp. 55-81.

San Nicolas, M. \& Rodriguez, R. (2016). La plaça d'Àngel Pestaña, el cor del barri de la Prosperitat. BTV Noticies. Cat.

Tamayo y Tamayo, M. (2004). El proceso de la investigación científica. Incluye evaluación y administración de proyectos de investigación. México: Limusa. 